\title{
Neighborhood Selection in Constraint-Based Local Search for Protein Structure Prediction
}

\author{
Swakkhar Shatabda ${ }^{1,2}$, MA Hakim Newton ${ }^{1}$, and Abdul Sattar ${ }^{1,2}$ \\ ${ }^{1}$ Institute for Intelligent and Integrated Systems (IIIS), Griffith University \\ ${ }^{2}$ Queensland Research laboratory, National ICT Australia(NICTA)
}

\begin{abstract}
Protein structure prediction (PSP) is a very challenging constraint optimization problem. Constraint-based local search approaches have obtained promising results in solving constraint models for PSP. However, the neighborhood exploration policies adopted in these approaches either remain exhaustive or are based on random decisions. In this paper, we propose heuristics to intelligently explore only the promising areas of the search neighborhood. On face centered cubic lattice using a realistic $20 \times 20$ energy model and standard benchmark proteins, we obtain structures with significantly lower energy and RMSD values than those obtained by the state-of-the-art algorithms.
\end{abstract}

\section{Introduction}

$A b$ initio methods for protein structure prediction (PSP), without using any templates or structures of known similar proteins, starts searching from the scratch for the native structure that has the minimum free energy. Due to the complexity of all-atomic detailed models and unknown factors of the energy function, the general paradigm of $a b$ initio PSP has been to begin with the sampling of a large set of candidate or decoy structures guided by a scoring function. In the final stage, the refinements [22] are done to achieve the realistic structure. Given a primary amino acid sequence of a protein, PSP can be defined as: find a self-avoiding walk on a discrete lattice that minimizes a contact-based energy function. However, the conformational search space still remains huge and the problem itself remains a very challenging constraint optimization problem.

Given a current partial or complete solution, selection of the neighboring solutions for further exploration is a key factor in the performance of constraint programming $(\mathrm{CP})$ and local search approaches. Neighborhood selections involves selection of variables and values. Unguided random selection [23, 28], costly exhaustive generation [5] and filtering or ordering techniques for enumeration [28, 19] are not much effective for neighborhood selection in PSP. In other domains, such as propositional satisfiability promising variables are selected using different variable selection strategies $[20,1]$.

In this paper, we propose several novel component fitness functions for neighborhood selection in PSP. These heuristics are an energy contribution function, a core-based distance function and a free lattice-neighbor count based function. 
These heuristics are derived from domain knowledge and are used along with a hint based variable selection strategy. The aggregate of these heuristics are used in selecting candidate structures. To the best of our knowledge, this is the first application of intelligent variable selection strategy in PSP. We also propose a new chain growth initialization for the energy model used. Experimental results show that our method significantly improves over the state-of-the-art algorithms and produces structures with lower energy and RMSD values for standard benchmark proteins on face centered cubic lattices.

\section{Related Work}

Simplified models such as Hydrophobic-Polar (HP) energy model [9] and discrete lattices have been studied extensively by many researchers within various frameworks such as constraint programming [7], genetic algorithms [21], and memory-based local search approaches [24].

In contrast to HP models, elaborate energy functions derived by using statistical methods $[14,2]$ take into consideration all $20 \times 20$ amino-acid interactions. Using secondary information and constraint programming techniques, Dal Palu et al. [19] developed a method to predict tertiary structures of real proteins. They also proposed several generalized and problem specific heuristics [17]. Later, they also developed a highly optimized constraint solver named COLA [18].

A two-stage optimization method was proposed in [27] by Ullah et al. It uses CPSP tool by Backofen et al. [12] to provide initial structure for local search procedure on FCC lattice and an elaborate energy function. The twostage optimization approach was reported to outperform simulated annealingbased local search procedure [25]. Ullah et al. also used large neighborhood search techniques on top of the COLA solver [28]. A fragment assembly method was proposed in [16] to produce low energy structures. Later, a filtering techniques for loop modeling [4] was proposed using CP techniques. In a recent work, several effective heuristics were used in a mixed fashion in [23] which produced stateof-the-art results on real proteins from standard benchmark set for contactbased energy models. Our work in this paper uses a hint-based variable selection strategy to generate the neighborhoods to be explored. Our work also uses a new chain growth initialization method.

Among other approaches in PSP are population based methods [8] and genetic algorithms [26] and CP techniques for side-chain models [13].

\section{The Problem Model and CP Formulation}

Proteins are polymers of amino-acid monomers. There are 20 different amino acids. In a simplified model, all monomers have an equal size and all bonds are of equal length. In a CP formulation, each monomer is modeled by a point in a three dimensional lattice (lattice constraint). The given amino acid sequence fits into the lattice: every pair of consecutive amino acids in the sequence are also neighbors in the lattice (chain constraint) and two monomers can not occupy the 
same point in the lattice (self avoiding constraint). A simplified energy function is used in calculating the energy of a structure.

Two lattice points $p, q \in \mathbb{L}$ are said to be in contact or neighbors of each other, if $q=p+\boldsymbol{v}_{\boldsymbol{i}}$ for some vector $\boldsymbol{v}_{i}$ in the basis of $L$. The Face Centered Cubic (FCC) lattice is preferred since it provides the densest packing [6] for spheres of equal size and the highest degree of freedom for placing an amino acid. The points in FCC lattice are generated by the following basis vectors: $\boldsymbol{v}_{\mathbf{1}}=(1,1,0), \boldsymbol{v}_{\mathbf{2}}=(-1,-1,0), \boldsymbol{v}_{\mathbf{3}}=(-1,1,0), \boldsymbol{v}_{\mathbf{4}}=(1,-1,0), \boldsymbol{v}_{\mathbf{5}}=(0,1,1)$, $\boldsymbol{v}_{\mathbf{6}}=(0,1,-1), \boldsymbol{v}_{\mathbf{7}}=(0,-1,-1), \boldsymbol{v}_{\mathbf{8}}=(0,-1,1), \boldsymbol{v}_{\mathbf{9}}=(1,0,1), \boldsymbol{v}_{\mathbf{1 0}}=(-1,0,1)$, $\boldsymbol{v}_{\mathbf{1 1}}=(-1,0,-1), \boldsymbol{v}_{\mathbf{1 2}}=(1,0,-1)$. In FCC lattice, each point has 12 neighbors and distance between two neighbors is $\sqrt{2}$.

In our CP model, we are given a sequence $S$, where each element $s_{i} \in S$ is an amino-acid type. Each amino acid $i$ is associated with a point $p_{i}=\left(x_{i}, y_{i}, z_{i}\right) \in$ $\mathbb{Z}^{3}$. The decision variables are the $x, y$ and $z$ co-ordinates of a point. For a sequence of length $n$, the domain of the variables is the range $[-n, n]$. Formally, $\forall_{i} x_{i} \in[-n, n], \forall_{i} y_{i} \in[-n, n]$ and $\forall_{i} z_{i} \in[-n, n]$. The first point is assigned as $(0,0,0)$, which is a valid point in the FCC lattice. The rest of the points follows the constraint, $\forall_{i<n}\left(\boldsymbol{a}_{\boldsymbol{i}}\right) \in\left\{\boldsymbol{v}_{\mathbf{1}}, \cdots, \boldsymbol{v}_{\mathbf{1 2}}\right\}$. Here, $\boldsymbol{a}_{\boldsymbol{i}}$ is the absolute vector between points $\left(x_{i+1}, y_{i+1}, z_{i+1}\right)$ and $\left(x_{i}, y_{i}, z_{i}\right)$, and $\left\{\boldsymbol{v}_{\mathbf{1}}, \cdots, \boldsymbol{v}_{\mathbf{1 2}}\right\}$ are the basis vectors for FCC lattice. Thus all points satisfy the lattice constraint and chain constraint. The self-avoiding constraint is defined using the all-different constraint all-different $\left(\forall_{i} p_{i}\right)$. We define sqrdist $(i, j)$ as the square of Euclidean distances between two points $p_{i}$ and $p_{j}$. Now, $\operatorname{contact}(i, j)=1$, if $\operatorname{sqrdist}(i, j)=2$; and $\operatorname{contact}(i, j)=0$, if $\operatorname{sqrdist}(i, j) \neq 2$. For any given protein sequence $S$, the energy of a structure $c$ is defined as:

$$
E(c)=\sum_{j \geq i+1}^{n} \operatorname{contact}(i, j) \cdot \operatorname{energy}\left(s_{i}, s_{j}\right)
$$

where energy $\left(s_{i}, s_{j}\right)$ is the empirical energy value between two amino-acids of type $s_{i}$ and $s_{j}$ obtained from the energy matrix given in [2]. Given this model, PSP can be defined as follows: given a sequence $S$ of length $n$, find a self-avoiding walk $p_{1}, \cdots, p_{n}$ on the lattice that minimizes the energy i.e. obj $=E(c)$.

\section{Our Approach}

Our approach is based on component heuristic functions that are used with a hint based variable selection strategy and aggregate functions which are used for candidate selection. Rest of the section describes necessary details.

\subsection{Search Procedure}

The search starts with the greedy chain growth initialization procedure that produces a compact low energy structure. Based on a walk probability $w p$ (initially set to $5 \%$ ), a variable is selected randomly or from a hint based priority queue 
with tabu on recently selected variables and the corresponding amino-acid position is determined. Neighborhood is generated only for the position selected using a set of operators. Then the generated candidate moves are simulated. Simulation of a move temporarily calculates the changes in the heuristic functions without committing the move. At each iteration, one of the heuristics are selected randomly by a uniform random distribution. After simulation, the best candidate move is selected and executed. The execution updates all cost functions, constraints and propagate hints. Ties are broken using a uniform random distribution. The search keep tracks of the global minimum found and restarts from the last found global minimum whenever it gets stuck. Stagnation is determined by a number of non-improving moves from the last found global minimum. At stagnation, the stagnation parameter $s p$ (initially set to 500) and the walk probability $w p$ are multiplied by a factor (set 1.2). Parameters $w p$ and $s p$ are set to initial values, whenever a new global minimum is found. Pseudo-code of our algorithm is given in Algorithm 1.

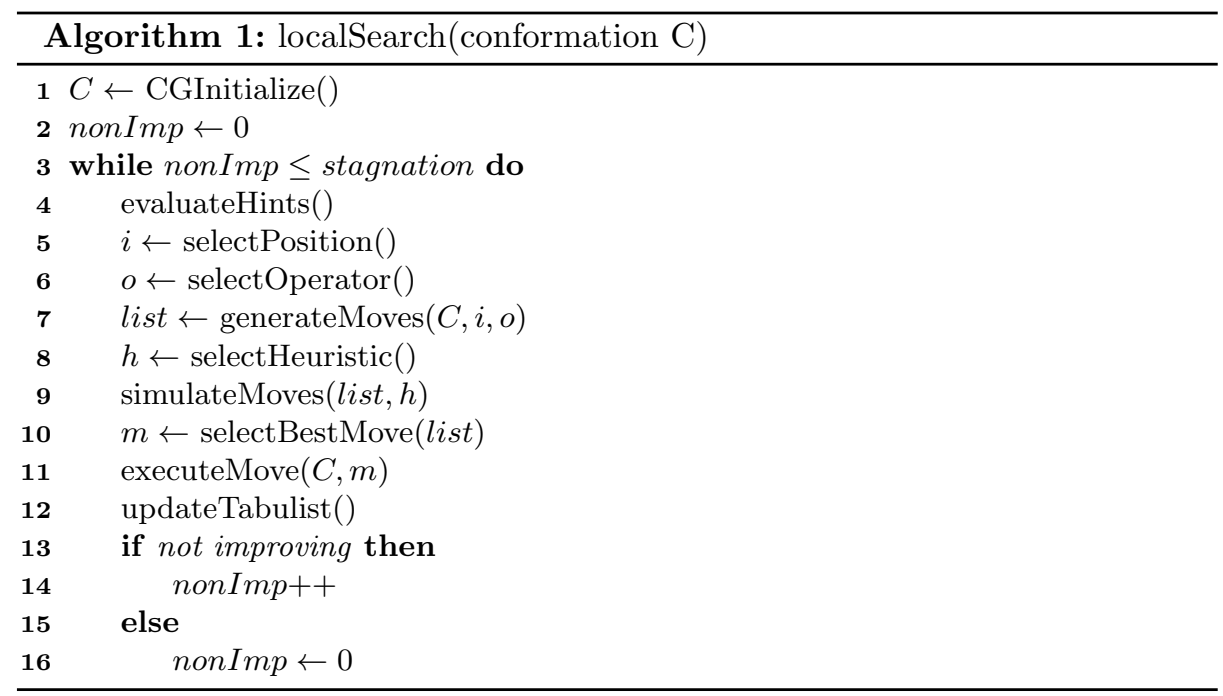

For a clear outline of our contribution, it is worthwhile to note the differences of our algorithm from the algorithm in [23]. In Line 1 of Algorithm 1, we call our new initialization function while the initialization in [23] uses a structure fully contained within a sphere. In Line $4-5$, we compute the hint heuristics and based on them, we select only one point, where the operators are applied. In contrast, in [23], points are selected randomly and then operators are applied in all selected points. 


\subsection{Heuristic Functions}

The empirical energy model gives an elaborate interaction energy contribution for the amino-acid types. The points with lower contribution to the total energy functions are preferred for selection. First, we define the first component fitness function that calculates the contribution of a point to the total energy of the structure. Formally,

$$
\operatorname{contr}(i)=\sum_{0 \leq k \leq n,|i-k|>1} \operatorname{contact}(i, k) \times \operatorname{energy}\left(s_{i}, s_{k}\right)
$$

The intuition is to select the variables with the maximum contr $(i)$ so that the corresponding operators can lower the energy contribution. Note that, the lowest energy contributions are generally negative. However, heuristics designed from domain knowledge often provide interesting insights. One such properties of protein folding is due to the solvent type water. This property lets the hydrophobic residues buried inside the structure and helps form a compact core. Based on this fact, several methods and heuristics have been developed for HP model $[12,7]$. However, interactions between two hydrophobic residues in contact in the core may result in repulsion (positive empirical energy) rather than attraction (negative empirical energy) and form a non-stable structure. For this reason, we don't use those heuristics directly into our model. We divide the 20 different aminoacids into two groups according to their similarity in interaction energy within each group. We run a simple $k$-means clustering algorithm on the empirical energy matrix to obtain two such groups (Group I: Ala, Phe, Gly, Ile, Leu, Met, Pro, Val, Trp, Tyr; Group II: Cys, Asp, Glu, His, Lys, Asn, Gln, Arg, Ser, Thr) such that interaction within each group minimizes the total energy contribution. We call these residues, affine residues and define an affine core, $a_{c}=\left(x_{c}, y_{c}, z_{c}\right)$, such that, $x_{c}=\frac{1}{|A|} \sum_{k \in A} x_{k}, y_{c}=\frac{1}{|A|} \sum_{k \in A} y_{k}, z_{c}=\frac{1}{|A|} \sum_{k \in A} z_{k}, A$ is the set of affine amino acid positions and $|A|$ denotes the total number of affine positions. Now we expect to move the affine amino acids towards the core. We define, our next component fitness function:

$$
\text { sqrdist-acore }(i)=\operatorname{sqrdist}\left(i, a_{c}\right)
$$

Naturally, we wish to move the distant affine positions nearer to the affine core, $a_{c}$, i.e. we wish to select the variables with maximum sqrdist-acore $(i)$ value. However, these component fitness functions to select the variable do not work well if there is not enough free positions in the lattice neighborhood of the point assigned to the position. Therefore, we define another component fitness function, free $(i)$. It counts the total number of free neighbors of a point $i$ in a lattice. Formally,

$$
\text { free-count }(i)=\sum_{k \in N(i)} \text { free }(k)
$$

Here, free $(k)=1$, if $k$ is free; and free $(k)=0$, if $k$ is occupied and $N(i)$ is set of neighbors of $i$ in the lattice. We wish to select the variables with the maximum 
free $(i)$ so that the number of possible moves become higher. It is interesting to note that we wish to select variables that maximizes all three component functions. Now, we aggregate the component fitness functions for all amino acid position $i$ and derive heuristic functions for the selection of candidate structures. We denote the heuristics as follows: the energy heuristic $h_{E}=\sum_{i \leq n} \operatorname{contr}(i)$, the affine core heuristic $h_{A}=\sum_{i \leq n}$ sqrdist-acore $(i)$ and the compactness heuristic $h_{F}=\sum_{i \leq n}$ free-count $(i)$. We wish to guide our search using these aggregate heuristic functions. The idea of component heuristics are previously used along with extremal optimization [11]. However, the heuristics used in this paper for the given energy model are novel themselves.

\subsection{Hint Based Variable Selection}

Now, we define two important terms: metric and hint. Each function $f\left(p_{1}, \cdots, p_{n}\right)$ has the parameters $p_{j}$ s that are either variables or other functions. A function $f$ depends on a variable $x$, denoted by $f \rightarrow x$, if $x$ is itself a parameter of $f$ or $f$ has a parameter $p \rightarrow x$. Each function $f$ has a non-negative metric $f_{m}$ denoting its evaluation. For each $x \leftarrow f$, it also has a non-negative hint $f_{h}(x)$ denoting the preference of changing $x$ 's value to improve $f_{m}$. A constraint $f$ is satisfied when $f^{m}=0$ and in that case $f_{h}(x)=0$ for any $x$, which means a constraint's metric improves when it is minimized.

In our model, all the component fitness functions are defined as constraints over the variables. For example, $\operatorname{contact}(i, j)$ is defined as a constraint, $\operatorname{sqrdist}(i, j)=$ 2, which is satisfied only when the square of Euclidean distance between $i$ and $j$ is equal to 2 . This function depends on the variables, $x_{i}, y_{i}, z_{i}$ and $x_{j}, y_{j}, z_{j}$. The metric of the function is simply the evaluation of the constraint that tests equality with 2 . If this constraint is not satisfied then the violation is added as the hint of these variables. Thus, variable violations for all the functions which are dependent on a particular variable is added as hint for that variable. Looking at these hint values, we decide which variable is to be selected in order to minimize the violation of the constraints. In our $\mathrm{CP}$ model, we take aggregate of all the component functions and take a summation of those aggregate functions to define another function on top as the hint provider. The variable violation for that top function is distributed as hints among the variables corresponding to amino acid positions. A simple heap or priority queue data structure with hint values is sufficient for us to decide which variable to select. We also maintain a tabu list to prevent recent variables to be selected. We use three different heuristics $h_{E}, h_{A}$ and $h_{F}$, and sum them to form a top function that provides the hints for all the variables.

\subsection{Chain Growth Initialization}

The procedure is inspired from chain growth algorithms previously applied to HP models [3]. The initialization starts by assigning $(0,0,0)$ to the first amino acid position. The rest of the variables are assigned following a greedy strategy. One of the free neighbors of the last assigned amino acid position $i-1$ is assigned 
to position $i$, such that the the assignment minimizes the partial objective function, obj $_{i}=\sum_{k<i} \operatorname{contact}(i, k) \times \operatorname{energy}\left(s_{i}, s_{k}\right)+$ free-count $(i) \times E_{U}[i]$. Here, $k$ is iterated over already assigned amino acid positions and $E_{U}[i]$ is the per-contact expected or average energy contribution of $i$ with possible unassigned amino acid positions in the chain. This partial objective function, $o b j_{i}$ is equal to the partial energy contribution, partial- $\mathrm{E}\left(p_{1}, \cdots, p_{i}\right)$. The tie-breaking is done according to a pre-defined order. Pseudo-code for variable selection for each step is given in Algorithm 3. The initialization procedure backtracks whenever, it fails to assign valid points to an amino acid position. This method guarantees to produce valid structure with low energy value. Pseudo-code of the chain growth initialization procedure is given in Algorithm 2.

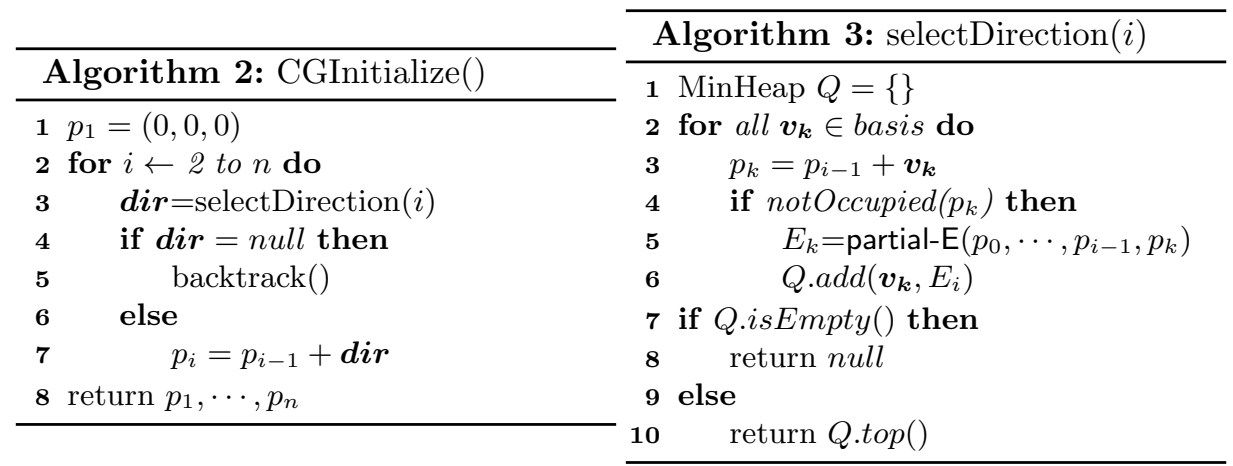

\subsection{Operators}

After a variable is selected, we can decide which amino acid position it corresponds to and apply the operators to that position. We make use of four types of operators (see Fig. 1). First two are jump move [23] and pull move [10] which are also used in the literature. We propose a single point pull move (Fig. 1(c)) similar to that of two point pull move and a single point push move which reverses the action of a single point pull move (Fig. 1(d)).

\subsection{Implementation}

We implemented our algorithm using $\mathrm{C}++$ on top of the constraint based local search (CBLS) system, Kangaroo [15]. The functions and the constraints are defined using invariants in Kangaroo. Invariants are special constructs that are defined by using mathematical operators over the variables. Propagation of hints, simulation of moves, execution and related calculations are performed incrementally by Kangaroo. 


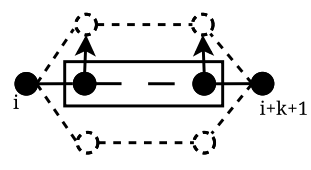

(a)

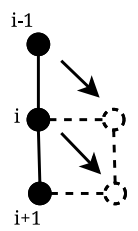

(b)

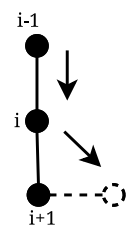

(c)

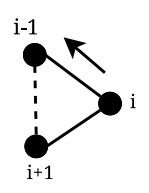

(d)

Fig. 1. Different Operators used: (a) jump moves $(\mathrm{k}=1,2,3)$ (b) two point pull move (c) single point pull move and (d) push move

\begin{tabular}{|c|c|c|c|c|c|c|c|c|c|c|c|}
\hline \multirow{3}{*}{$\begin{array}{c}\text { seq } \\
\text { no }\end{array}$} & \multirow{3}{*}{$\begin{array}{c}\text { pdb } \\
\text { id }\end{array}$} & \multirow{3}{*}{$\begin{array}{l}\text { seq } \\
\text { len }\end{array}$} & \multicolumn{3}{|c|}{ Our Approach } & \multicolumn{3}{|c|}{ Mixed [23] } & \multicolumn{3}{|c|}{ Hybrid [28] } \\
\hline & & & \multicolumn{2}{|c|}{ energy level } & \multirow{2}{*}{$\begin{array}{l}\text { avg } \\
\text { rmsd }\end{array}$} & \multicolumn{2}{|c|}{ energy level } & \multirow{2}{*}{$\begin{array}{c}\text { avg } \\
\text { rmsd }\end{array}$} & \multicolumn{2}{|c|}{ energy level } & \multirow{2}{*}{$\begin{array}{c}\text { avg } \\
\text { rmsd }\end{array}$} \\
\hline & & & best & avg & & best & avg & & best & avg & \\
\hline 1 & $4 \mathrm{rxn}$ & 54 & -168.78 & -164.28 & 5.56 & -165.21 & -156.32 & 6.29 & -157.70 & -140.13 & 9.99 \\
\hline 2 & 1enh & 54 & -158.74 & -152.43 & 5.33 & -158.75 & -146.69 & 6.61 & -154.24 & -141.99 & 10.04 \\
\hline 3 & $4 p t i$ & 58 & -221.59 & -205.42 & 5.86 & -219.52 & -198.42 & 7.07 & -213.70 & -196.23 & 11.92 \\
\hline 4 & 2igd & 61 & -187.96 & -179.36 & 6.34 & -187.20 & -174.19 & 9.33 & -184.29 & -157.20 & 13.30 \\
\hline 5 & 1ура & 64 & -257.02 & -248.47 & 6.12 & -249.90 & -239.98 & 7.53 & -221.11 & -208.10 & 13.42 \\
\hline 6 & 1r69 & 69 & -223.12 & -210.15 & 5.78 & -213.04 & -204.17 & 6.47 & -180.62 & -165.11 & 14.78 \\
\hline 7 & $1 \mathrm{ctf}$ & 74 & -230.86 & -220.04 & 6.14 & -224.29 & -213.81 & 7.23 & -204.88 & -195.23 & 12.65 \\
\hline 8 & $3 \mathrm{mx} 7$ & 90 & -332.79 & -321.58 & 6.58 & -328.12 & -311.56 & 8.18 & - & - & - \\
\hline 9 & $3 \mathrm{nbm}$ & 108 & -431.90 & -415.02 & 6.28 & -418.60 & -401.99 & 8.58 & - & - & - \\
\hline 10 & $3 \mathrm{mqo}$ & 120 & -476.06 & -464.57 & 6.46 & -465.74 & -455.27 & 8.86 & - & - & - \\
\hline 11 & $3 \mathrm{mr0}$ & 142 & -446.31 & -435.69 & 7.32 & -445.33 & -430.28 & 10.02 & - & - & - \\
\hline 12 & $3 p n x$ & 160 & -603.78 & -585.97 & 6.84 & -601.23 & -571.13 & 9.38 & - & - & - \\
\hline
\end{tabular}

Table 1. Results obtained by different algorithms for 12 proteins

\section{$5 \quad$ Experiments}

We ran our experiments on a cluster of computers with nodes equipped with Intel Xeon CPU X5650 processors @2.67GHz, QDR 4 x InfiniBand Interconnect. We compare our results with the mixed heuristic approach in [23] and the hybrid approach in [28]. All the algorithms are given 1 hour to finish each run, and the best and average energy levels of 50 runs are reported in Table 1 for 12 benchmark proteins. These proteins are also used in [23]. The blank values in the table are the cases where hybrid approach failed to produce any valid structure within the time limit. PDB ids, sequence length and average RMSD values are also reported in the table. The best values are indicated in bold faced font. For all the 12 proteins, our approach achieves lower energy levels. The significance of these values are confirmed from the values reported in the rmsd colmun. For any given structure produced by an algorithm,

$$
\operatorname{RMSD}=\sqrt{\frac{\sum_{i=1}^{n-1} \sum_{j=i+1}^{n}\left(\operatorname{dist}(i, j)^{\text {given }}-\operatorname{dist}(i, j)^{\text {native }}\right)^{2}}{n *(n-1) / 2}}
$$


Here dist $(i, j)$ is the Euclidean distance between two points $p_{i}$ and $p_{j}$. In calculating the RMSD values, the distance between two neighbors in the lattice is considered to be equal to the average distance $(3.8 \AA)$ between two $\alpha$-Carbons on the native structure. From the values reported we see, our approach also significantly improves over the other approaches in terms of the RMSD values; the lower the RMSD score, the better the performance. These values are significant since backbone reconstruction and addition of side-chain atoms can guarantee

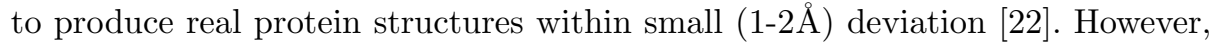
since lattice configurations can only approximate the positions of the amino acids in the real space, lower RMSD values produced by our algorithm are satisfactory.

\begin{tabular}{|c|l|c|c|c|c|c|c|}
\hline seq no & $\neg$ hint & $+\mathbf{h i n t}\left(\mathbf{h}_{\mathbf{A}}\right)$ & $+\mathbf{h i n t}\left(\mathbf{h}_{\mathbf{E}}\right)$ & $\neg \mathbf{h i n t}\left(\mathbf{h}_{\mathbf{F}}\right)$ & $\neg \mathbf{h}_{\text {select }}$ & init $_{\mathbf{r}}$ & all \\
\hline 1 & -146.61 & -149.61 & -151.25 & -158 & -156.08 & -136.23 & $\mathbf{- 1 6 4 . 2 8}$ \\
2 & -136.35 & -137.62 & -128.15 & -147.03 & -142.44 & -137.08 & $\mathbf{- 1 5 2 . 4 3}$ \\
3 & -185.52 & -192.15 & -189.45 & -194.62 & -200.6 & -197.88 & $\mathbf{- 2 0 5 . 4 2}$ \\
4 & -165.5 & -158.16 & -157.64 & -171.24 & -164.12 & -149.74 & $\mathbf{- 1 7 9 . 3 6}$ \\
5 & -235.75 & -235.45 & -236.88 & -244.44 & -244.71 & -219.91 & $\mathbf{- 2 4 8 . 4 7}$ \\
6 & -193.87 & -187.64 & -180.61 & -183.20 & -182.85 & -178.25 & $\mathbf{- 2 1 0 . 1 5}$ \\
7 & -197.83 & -204.48 & -198.45 & -210.27 & -206.84 & -182.46 & $\mathbf{- 2 2 0 . 0 4}$ \\
\hline
\end{tabular}

Table 2. Average energy level achieved by different variants of our algorithm

In order to test the effectiveness of different components of our approach, we ran different variants of our approach on first 7 proteins and report average energy level of 20 runs for each of them in Table 2. First, we report the performance of a variant without using the hint based variable selection in column $\neg$ hint. It shows how the hint based system can improve on this variant to achieve the final performance shown in 'all' column. The 'all' column, for convenience of the reader, again shows the average energy values obtained by our approach (as shown in Table 1). Then we ran two variants with hints for $h_{A}$ only (column $\left.+\operatorname{hint}\left(h_{A}\right)\right)$ and with hints for $h_{E}$ only (column $\left.+\operatorname{hint}\left(h_{E}\right)\right)$. These two variants show the effectiveness of using these two heuristics as hint provider individually. We see that these two heuristics are showing better performance than the hintless variant for most of the proteins, and $+\operatorname{hint}\left(h_{A}\right)$ is performing better than $+\operatorname{hint}\left(h_{E}\right)$. It reveals that the energy function itself is not enough for providing hints for the search and heuristic approximations can actually improve the performance. However, both of them shows effectiveness of hints over the no-hint variant. Then, we ran another variant with hints for both $h_{E}$ and $h_{A}$, but not using hints for $h_{F}$ (column $\neg \operatorname{hint}\left(h_{F}\right)$ ). This particular variant shows the combined performance of two heuristics as hint provider and also shows the relative strength of the other hint heuristic $h_{F}$ that is absent. We get a clear idea of the strength of $h_{F}$ by comparing it with the final results in column 'all' and also strength of the combined variant by comparing the results with the individual columns of $+\operatorname{hint}\left(h_{A}\right)$ and $+\operatorname{hint}\left(h_{E}\right)$. We see that the combination of $h_{E}$ and $h_{A}$ which is $\neg \operatorname{hint}\left(h_{F}\right)$ works better than the individual variants. 
However, its only after adding the hints for $h_{F}$, its possible to achieve the final performance. Another variant uses the hint based system but the selection of candidate structures is guided by $h_{E}$ only (column $\neg h_{\text {select }}$ ). This indicates the effectiveness of the heuristics to select candidate structures. The penultimate column ( init $_{r}$ ) shows the results achieved by replacing our chain growth algorithm by a random initialization. From the reported values we see that chain growth initialization has a greater impact on most of the proteins with respect to the random initialization methods.

To show how the search makes progress, we plot log of average energy levels achieved, (for convenience of display in the chart) added by a threshold of 250 for the protein 4pti against iteration count in Fig. 2. We see that the variant without hint ( $\neg$ hint) works worst. It improves only at the beginning and then gets stagnant. Variants with hints for $h_{E}$ and $h_{A}$ works better than this. If we add hints for all the heuristic functions but guide the search with $h_{E}$ only $\left(\neg h_{\text {select }}\right)$, it can further improve. However, the best performance is only after we use all the heuristics to guide the search. In case of random initialization, we find that it can gradually improve, but most of the time is spent to achieve the initial level that is achieved by the chain growth initialization for other variants.

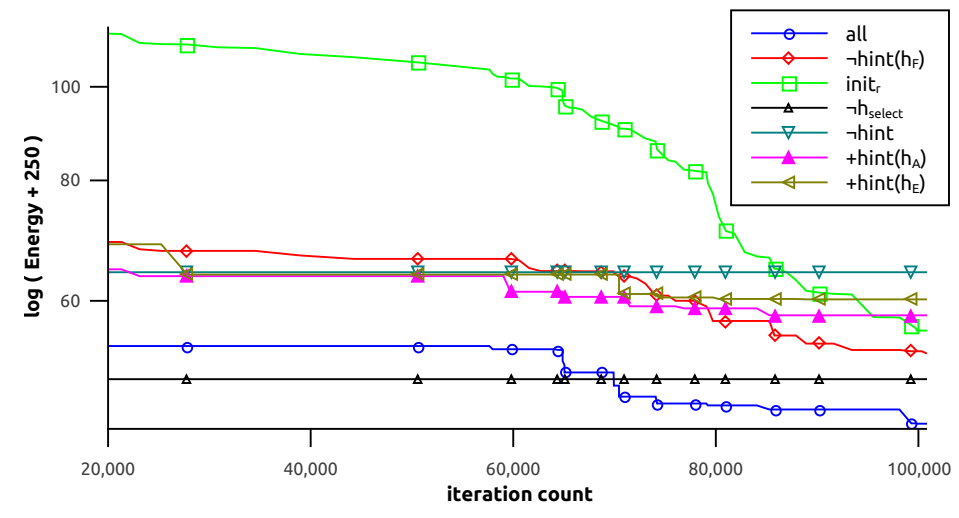

Fig. 2. Search progress of different variants for the protein 4 pti.

\section{Conclusion}

In this paper, we have proposed an intelligent variable selection strategy for candidate generation in local search methods for protein structure prediction problem using $20 \times 20$ energy model on face centered cubic lattice. In addition to this, we also have proposed a new chain growth initialization procedure and heuristic functions to select variables and candidates at each iteration. Our method 
significantly improves over the state-of-the-art algorithms. In future, we wish to explore the strength of our proposed scheme on other domains and on other models of protein structure prediction as well.

\section{Acknowledgments}

We gratefully acknowledge the support of the Griffith University eResearch Services Team and the use of the High Performance Computing Cluster "Gowonda" to complete this research and NICTA, which is funded by the Australian Government as represented by the Department of Broadband, Communications and the Digital Economy and the Australian Research Council through the ICT Centre of Excellence program.

\section{References}

1. Balint, A., Fröhlich, A.: Improving stochastic local search for sat with a new probability distribution. In: Proceedings of the 13th international conference on Theory and Applications of Satisfiability Testing. pp. 10-15. SAT'10, Springer-Verlag, Berlin, Heidelberg (2010)

2. Berrera, M., Molinari, H., Fogolari, F.: Amino acid empirical contact energy definitions for fold recognition in the space of contact maps. BMC Bioinformatics 4, 8 (2003)

3. Bornberg-Bauer, E.: Chain growth algorithms for hp-type lattice proteins. In: Proceedings of the first annual international conference on Computational molecular biology. pp. 47-55. RECOMB '97, ACM, New York, NY, USA (1997)

4. Campeotto, F., Palù, A.D., Dovier, A., Fioretto, F., Pontelli, E.: A filtering technique for fragment assembly- based proteins loop modeling with constraints. In: CP. pp. 850-866 (2012)

5. Cebrián, M., Dotú, I., Van Hentenryck, P., Clote, P.: Protein structure prediction on the face centered cubic lattice by local search. In: Proceedings of the 23rd national conference on Artificial intelligence - Volume 1. pp. 241-246. AAAI'08, AAAI Press (2008)

6. Cipra, B.: Packing challenge mastered at last. Science 281(5381), 1267 (1998)

7. Dotu, I., Cebrian, M., Van Hentenryck, P., Clote, P.: On lattice protein structure prediction revisited. Computational Biology and Bioinformatics, IEEE/ACM Transactions on 8(6), 1620-1632 (nov-dec 2011)

8. Kapsokalivas, L., Gan, X., Albrecht, A.A., Steinhöfel, K.: Population-based local search for protein folding simulation in the MJ energy model and cubic lattices. Computational Biology and Chemistry 33(4), 283-294 (2009)

9. Lau, K.F., Dill, K.A.: A lattice statistical mechanics model of the conformational and sequence spaces of proteins. Macromolecules 22(10), 3986-3997 (1989)

10. Lesh, N., Mitzenmacher, M., Whitesides, S.: A complete and effective move set for simplified protein folding. In: Proceedings of the seventh annual international conference on research in computational molecular biology. pp. 188-195. RECOMB '03, ACM, New York, NY, USA (2003)

11. Lu, H., Yang, G.: Extremal optimization for protein folding simulations on the lattice. Comput. Math. Appl. 57, 1855-1861 (June 2009)

12. Mann, M., Will, S., Backofen, R.: CPSP-tools - Exact and complete algorithms for high-throughput 3 D lattice protein studies. Bmc Bioinformatics 9(1), 230 (2008) 
13. Mann, M., Hamra, M.A., Steinhöfel, K., Backofen, R.: Constraint-based local move definitions for lattice protein models including side chains. In: Proceedings of the Fifth Workshop on Constraint Based Methods for Bioinformatics (WCB09) (2009)

14. Miyazawa, S., Jernigan, R.L.: Estimation of effective interresidue contact energies from protein crystal structures: quasi-chemical approximation. Macromolecules 18(3), 534-552 (Mar 1985)

15. Newton, M., Pham, D.N., Sattar, A., Maher, M.J.: Kangaroo: An efficient constraint-based local search system using lazy propagation. In: CP. pp. 645-659 (2011)

16. Palù, A.D., Dovier, A., Fogolari, F., Pontelli, E.: Exploring protein fragment assembly using CLP. In: IJCAI. pp. 2590-2595 (2011)

17. Palù, A.D., Dovier, A., Pontelli, E.: Heuristics, optimizations, and parallelism for protein structure prediction in CLP $(F D)$. In: PPDP. pp. 230-241 (2005)

18. Palù, A.D., Dovier, A., Pontelli, E.: A constraint solver for discrete lattices, its parallelization, and application to protein structure prediction. Softw. Pract. Exper. 37, 1405-1449 (November 2007)

19. Palù, A.D., Will, S., Backofen, R., Dovier, A.: Constraint based protein structure prediction exploiting secondary structure information. In: Proceedings of Italian Conference on Computational Logic. CLIC'04 (2004)

20. Pham, D.N., Thornton, J., Gretton, C., Sattar, A.: Advances in local search for satisfiability. In: Proceedings of the 20th Australian joint conference on Advances in artificial intelligence. pp. 213-222. AI'07, Springer-Verlag, Berlin, Heidelberg (2007)

21. Rashid, M.A., Hoque, M.T., Newton, M., Pham, D.N., Sattar, A.: A new genetic algorithm for simplified protein structure prediction. In: Proceedings of the 25th Australian Joint Conference on Artificial Intelligence. AI'12, Sydney, NSW, Australia (2012)

22. Rotkiewicz, P., Skolnick, J.: Fast procedure for reconstruction of full-atom protein models from reduced representations. Journal of Computational Chemistry 29(9), 1460-1465 (2008)

23. Shatabda, S., Newton, M.A.H., Sattar, A.: Mixed heuristic local search for protein structure prediction. In: Proceedings of the Twenty-Seventh AAAI Conference on Artificial Intelligence, AAAI 2013, Bellevue, Washington, USA, July 14-18, 2013. AAAI Press (2013)

24. Shatabda, S., Newton, M., Rashid, M.A., Pham, D.N., Sattar, A.: The road not taken: retreat and diverge in local search for simplified protein structure prediction. BMC Bioinformatics 14(2), 1-9 (2013)

25. Steinhofel, K., Skaliotis, A., Albrecht, A.: Relating time complexity of protein folding simulation to approximations of folding time. Computer Physics Communications 176(7), 465-470 (2007)

26. Torres, S.R.D., Romero, D.C.B., Vasquez, L.F.N., Ardila, Y.J.P.: A novel ab-initio genetic-based approach for protein folding prediction. In: Proceedings of the 9th annual conference on Genetic and evolutionary computation. pp. 393-400. GECCO '07, ACM, New York, NY, USA (2007)

27. Ullah, A.D., Kapsokalivas, L., Mann, M., Steinhöfel, K.: Protein folding simulation by two-stage optimization. In: Computational Intelligence and Intelligent Systems, pp. 138-145. Springer (2009)

28. Ullah, A.D., Steinhöfel, K.: A hybrid approach to protein folding problem integrating constraint programming with local search. BMC Bioinformatics 11(S-1), 39 (2010) 